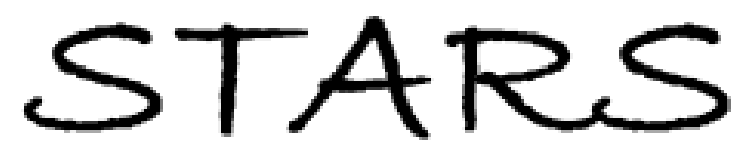

University of Central Florida

STARS

$1-1-2003$

\title{
Tunable electronic lens using a gradient polymer network liquid crystal
}

Hongwen Ren

University of Central Florida

Shin-Tson Wu

University of Central Florida

Find similar works at: https://stars.library.ucf.edu/facultybib2000 University of Central Florida Libraries http://library.ucf.edu

This Article is brought to you for free and open access by the Faculty Bibliography at STARS. It has been accepted for inclusion in Faculty Bibliography 2000 s by an authorized administrator of STARS. For more information, please contactSTARS@ucf.edu.

\section{Recommended Citation}

Ren, Hongwen and Wu, Shin-Tson, "Tunable electronic lens using a gradient polymer network liquid crystal" (2003). Faculty Bibliography 2000s. 3984.

https://stars.library.ucf.edu/facultybib2000/3984

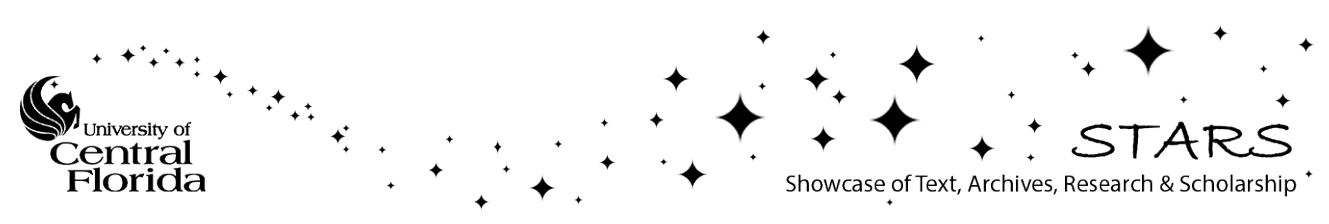




\title{
Tunable electronic lens using a gradient polymer network liquid crystal
}

Cite as: Appl. Phys. Lett. 82, 22 (2003); https://doi.org/10.1063/1.1534915

Submitted: 15 October 2002 . Accepted: 12 November 2002 . Published Online: 30 December 2002

\author{
Hongwen Ren, and Shin-Tson Wu
}

\section{ARTICLES YOU MAY BE INTERESTED IN}

Tunable-focus flat liquid crystal spherical lens

Applied Physics Letters 84, 4789 (2004); https://doi.org/10.1063/1.1760226

Tunable Fresnel lens using nanoscale polymer-dispersed liquid crystals

Applied Physics Letters 83, 1515 (2003); https://doi.org/10.1063/1.1604943

Inhomogeneous nanoscale polymer-dispersed liquid crystals with gradient refractive index Applied Physics Letters 81, 3537 (2002); https://doi.org/10.1063/1.1519102

\section{Applied Physics Letters}

Mid-IR and THz frequency combs special collection 


\title{
Tunable electronic lens using a gradient polymer network liquid crystal
}

\author{
Hongwen Ren and Shin-Tson $\mathrm{Wu}^{\mathrm{a})}$ \\ School of Optics/CREOL, University of Central Florida, Orlando, Florida 32816
}

(Received 15 October 2002; accepted 12 November 2002)

Tunable electronic lenses using gradient polymer network liquid crystal (PNLC) cells were demonstrated. By changing the photomask pattern, both positive and negative lenses were fabricated. The advantages of such a PNLC lens are low operation voltage, large aperture size, and simple electrode design. To overcome the polarization dependence, stacking two orthogonal homogeneous PNLC cells is considered. (C) 2003 American Institute of Physics.

[DOI: $10.1063 / 1.1534915$ ]

Liquid crystal (LC) adaptive lenses are attractive for a variety of applications in machine vision, photonics and eye glasses. Several approaches have been developed for obtaining tunable focus LC lenses. ${ }^{1-9}$ The first type of LC lens is driven by an inhomogeneous electric field which leads liquid crystal to exhibit lens-like gradient refractive index distribution. ${ }^{1-6}$ This type of LC lens is often called microlens because it is difficult to scale up the lens aperture. The second type LC lens is driven by a homogeneous electric field. Examples include surface relief microlens, ${ }^{7}$ Fresnel diffraction lens, ${ }^{8}$ as well as polymer stabilized LC lens which was fabricated by using Gaussian-shaped laser beam. ${ }^{9}$ However, the second type lens is also difficult to scale up aperture. The major advantage of the homogeneous electric field approach is the simple driving electronics. The electrode is a continuous indium tin oxide (ITO) film, not discrete pixels. A uniform voltage is applied to the cell to generate refractive index gradient.

Recently, we have demonstrated a tunable LC prism and lens using a gradient refractive index nanoscale polymerdispersed liquid crystal (GRIN PDLC) device. ${ }^{10}$ The GRIN PDLC lens can have positive or negative focal length depending on the pattern of the photomask. Owing to the nanoscale LC droplets involved, the GRIN PDLC lenses are highly transparent in the whole visible region, independent of polarization, and has $\sim 100 \mu$ s response time. The shortcoming is that it requires a relatively high $\left(\sim 100 V_{\text {rms }}\right)$ operation voltage.

To lower the operation voltage, in this letter we present tunable negative and positive LC lenses using inhomogeneous polymer network liquid crystal (PNLC) cells. The gradient refractive index of the PNLC cells is formed by exposing an UV light through a patterned photomask. The higher UV exposure region results in a higher polymer concentration and smaller LC domains so that its threshold voltage is higher. ${ }^{11}$ When a uniform voltage is applied to the cell, the gradient LC director distribution is generated. In this approach, the LC concentration exceeds $95 \%$ so that the operation voltage is low.

Figure 1 illustrates the fabrication process of a negative PNLC lens. The key element is the patterned photomask. To obtain a PNLC cell with an inhomogeneous centrosymmetric

a)Electronic mail: swu@mail.ucf.edu polymer network profile, a circular continuously variable optical density filter (L32-700, Edmund Optics) was used as the photomask. The radius of the mask is $1.87 \mathrm{~cm}$. The optical density (OD) decreases outward radically from a maximum $\mathrm{OD}=1.0$ at the center to $\mathrm{OD} \sim 0.04$ at the edges. When the uniform UV light (Loctite model 98016) passes through the photomask, the output intensity varies in parabolic profile across the sample. The area with a stronger UV light would accelerate the polymerization process and produce a higher polymer concentration. Conversely, the area with a weaker UV exposure would have a lower polymer network concentration. As a result, a polymer network with inhomogeneous centrosymmetric morphology is formed.

This centrosymmetric PNLC morphology leads to a concave refractive index profile which functions as a negative lens. When a uniform electric field is applied, the LC directors are reoriented to different degrees. The area with a looser network packing (center) has a lower threshold voltage than that of a denser zone (edges). Thus, the concave refractive index is formed. As the applied voltage increases, the curvature of the gradient lens decreases. In the high voltage regime, nearly all the LC directors are aligned along the electric field direction. The gradient no longer exists and the lens effect vanishes.

In our experiments, we first fabricated a negative lens PNLC cell using the photomask shown in Fig. 1. We mixed $3 \%$ UV-curable monomer BAB6 (containing 2 wt \% photoinitiator IRG184) in a LC host (W-1331, $\Delta n=0.229)$. The BAB6 monomer has a common rod-like structure with a reactive double bond at both sides. The $\mathrm{LC} /$ monomer mixture was injected to a homogeneous LC cell composing of ITO-

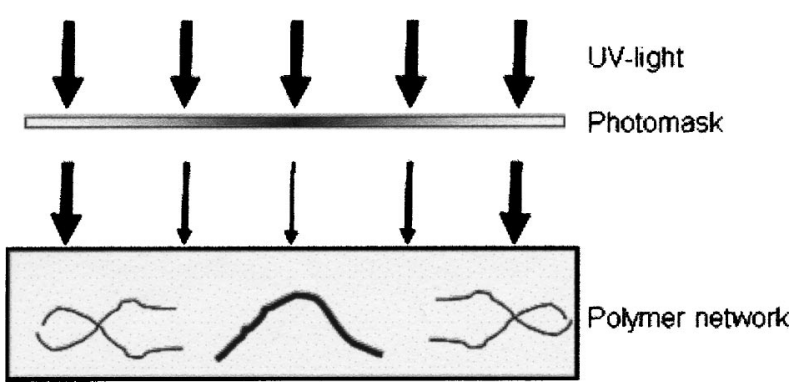

FIG. 1. Fabrication of an inhomogeneous PNLC using a centrosymmetric circular continuously variable density filter as photomask. $O D=1$ at center and 0.04 at edges. 


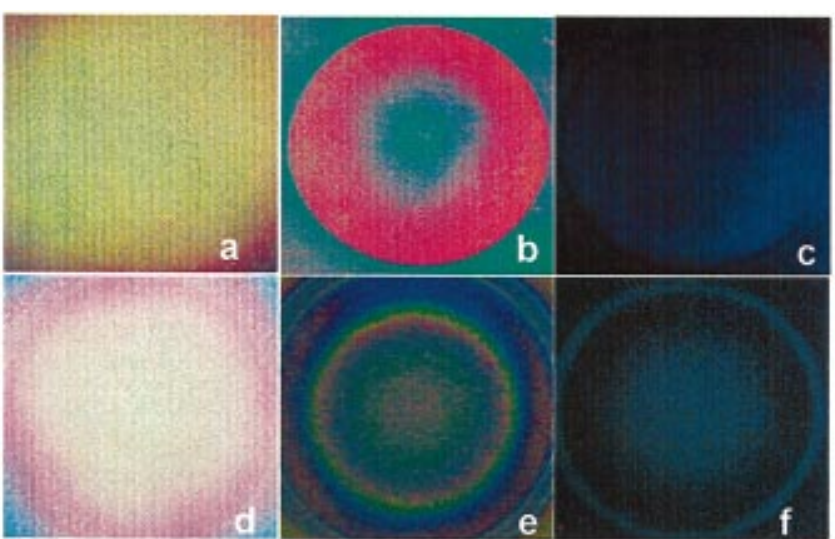

FIG. 2. (Color) Photographs of the two PNLC samples at different operation voltages: (a)-(c): $V=0,2.8$, and $30 V_{\text {rms }}$ for a negative lens; (d) $-(\mathrm{f}): V$ $=0,2$, and $35 V_{\text {rms }}$ for a positive lens. Polarizers are crossed. LC: W-1331 and cell gap $d=16.5 \mu \mathrm{m}$.

coated glass substrates. The inner surfaces of the ITO-glass substrates were coated with a thin polyimide layer. The cell gap of the homogeneous cells is $d=16.5 \mu \mathrm{m}$. A diaphragm with $2 \mathrm{~cm}$ diameter was put on top of the photomask. The measured average UV intensity behind the photomask is 14 $\mathrm{mW} / \mathrm{cm}^{2}$ and the exposure time is $60 \mathrm{~min}$.

The prepared PNLC sample is highly transparent in the voltage-off state. We first checked the gradient refractive index by observing the birefringence colors on a light table. The rubbing direction of the cell was oriented at $45^{\circ}$ with respect to the fast axis of the linear polarizer. The analyzer is crossed to the polarizer. Three photographs of the PNLC sample at $V=0,2.8$ and $30 V_{\text {rms }}$ were taken and results are shown in Figs. 2(a), 2(b), and 2(c), respectively.

At $V=0$, a relatively uniform yellowish color was observed in the exposed circle ( $2 \mathrm{~cm}$ diameter). Outside this area, the LC/monomer was partially cured by the scattered UV light. The color of the photos is due to the phase retardation of the PNLC cell. The threshold voltage of the PNLC cell is $V_{\mathrm{th}} \sim 1.5 V_{\mathrm{rms}}$. Above this threshold, the birefringence color changes with the applied voltage. In our experiment, we found that the color change starts from the center and gradually expands to the boarders. This implies that the central area has a lower threshold voltage than that of the edges. As mentioned before, the weaker UV exposure leads to a lower polymer concentration which results in a lower threshold voltage.

Figure 2(b) shows the birefringence color of the PNLC cell at $V=2.8 V_{\text {rms }}$. The central area clearly has a different color from the borders. This confirms the expected gradient phase distribution. The steepness of the phase (or color) change depends on the photomask design. As the applied voltage is sufficiently higher than the threshold, the bulk LC directors are reoriented perpendicular to the substrates. Under such a circumstance, the PNLC appears black between crossed polarizers, as shown in Fig. 2(c).

The inhomogeneous network size distribution is due to the phase separation process, which is different from the conventional PNLC. In the inhomogeneous PNLC, the polymerization rate starts preferentially from the border of the irradiated spot and propagates toward the center. The consumption of monomers in these regions lowers the
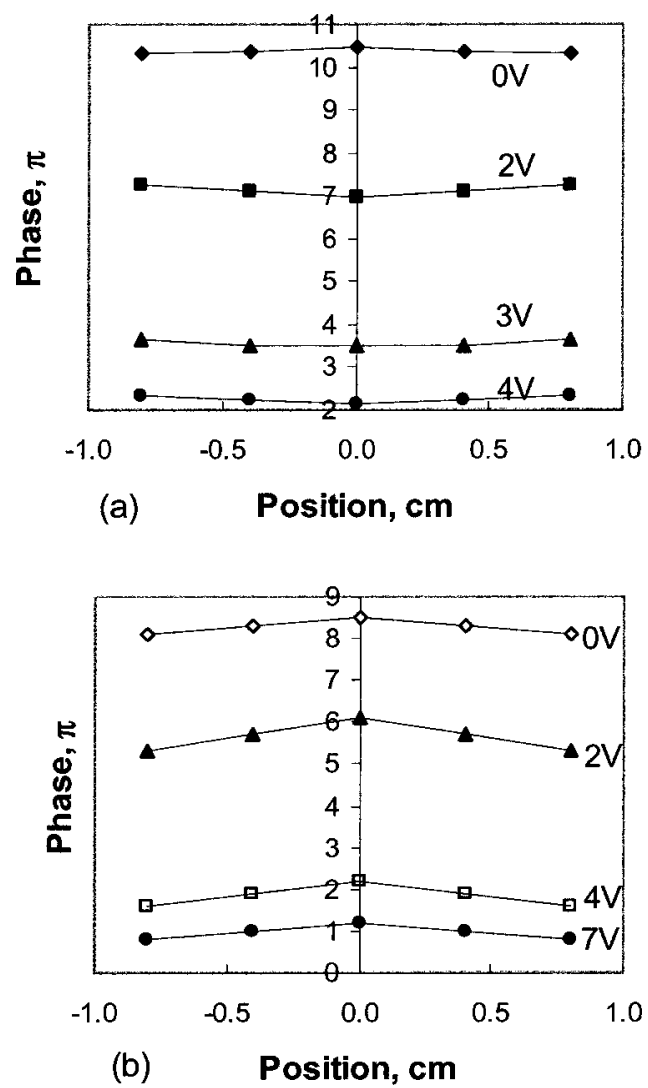

FIG. 3. Spatial phase profile of the negative (a) and positive (b) lenses at various voltages. LC: $\mathrm{W}-1331, d=16.5 \mu \mathrm{m}$.

chemical potential. This pushes the monomers to diffuse from low to high intensity region. On the contrary, the LC molecules diffuse from high to low intensity direction to balance the chemical potential. ${ }^{12}$ As a result, in an inhomogeneous PNLC sample, the network size and their concentration both exhibit gradient distributions. The LC-rich region has a slightly larger LC domain and higher LC concentration.

In order to quantitatively characterize the spatial phase profile of the inhomogeneous PNLC cell, we chose five spots along a diameter for measurements. One is at the center of the photopolymerization zone, two are at half-way to the boarders and two are near the borders. At each spot, we measured the voltage-dependent transmittance using a $\mathrm{He}-\mathrm{Ne}$ laser under the crossed and parallel polarizer conditions. From the measured transmittances, phase retardation was calculated. ${ }^{13}$ Results are depicted in Fig. 3(a).

In Fig. 3(a), a small but positive phase difference $(\Delta \delta$ $\left.=\delta_{\text {center }}-\delta_{\text {border }} \sim 0.2 \pi\right)$ is observed at $V=0$. This positive $\Delta \delta$ is mainly due to the cell gap nonuniformity. The cell gap at the center and boarder was measured to be 16.45 and $16.10 \mu \mathrm{m}$, respectively. This difference could explain the observed positive $\Delta \delta$. As the applied voltage increases, both $\delta_{\text {center }}$ and $\delta_{\text {border }}$ decrease and $\Delta \delta$ becomes negative due to the voltage-induced LC director reorientation. At $V$ $=2 V_{\text {rms }}, \Delta \delta \sim-0.32 \pi$ and the PNLC cell functions like a negative lens. As the applied voltage continues to increase, the phase gradient is gradually flattened. At $V \sim 30 V_{\mathrm{rms}}[\mathrm{Fig}$. 2(c)], $\Delta \delta$ is diminishing because the LC directors in the whole cell are aligned along the electric field direction.

To estimate the focal length $(F)$ of this negative lens, we choose the PNLC cell at $2 V_{\text {rms }}$ as an example. The effective 
focal length is related to the lens radius $r$, wavelength $\lambda$, and phase difference $\Delta \delta$ as: $F=\pi r^{2} / \lambda \Delta \delta$. In our experiment, $r$ $=0.8 \mathrm{~cm}, \lambda=0.633 \mu \mathrm{m}$, and $\Delta \delta=-0.32 \pi$. Thus, the calculated effective focal length is around $F \sim-310 \mathrm{~m}$. Such a long focus lens is still useful for astronomy applications. To shorten the focal length, it is necessary to sharpen the phase gradient, i.e., $\Delta \delta$. For example, we could enlarge the optical density difference between the center and the edges of the photomask, use a high birefringence LC mixture and optimize the monomer concentration.

By reversing the optical density pattern of the photomask depicted in Fig. 1, we then fabricated a tunable positive lens. The fabrication process of the cell remains the same except that the diameter of the irradiated circle is $1.7 \mathrm{~cm}$ and the monomer concentration is $5 \mathrm{wt} \%$. We also viewed the sample between crossed polarizers and took three photographs as shown in Figs. $2(\mathrm{~d}-\mathrm{f})$ at different voltages. At $V$ $=0$, the color was not very uniform. Again, this results from the cell gap nonuniformity. As the applied voltage exceeds the threshold, color starts to change from the borders and gradually extends to the center, like a swallowing spot. On the contrary, decreasing voltage changes the birefringence color from center to borders, like a vomiting spot. This cell acts as a positive lens.

The measured phase retardation of the cell is plotted in Fig. 3(b). At $2 V_{\text {rms }}, \Delta \delta=0.8 \pi$ which corresponds to a 120 $\mathrm{m}$ focal length. As the voltage increases, $\Delta \delta$ decreases so that the focal length increases. A too high monomer concentration would result in an increased light scattering and decreased phase change and is, therefore, not favorable.

Similar to a conventional PNLC, our PNLC lens is polarization dependent, i.e., it works for a linearly polarized light. To overcome this polarization dependence, we could stack two cells with orthogonal alignment directions. If the top cell is designed for the extraordinary ray, then the bottom cell would be for ordinary ray. Since the lens is a single pixel device, the pixel registration problem as occurred in a high resolution display is not relevant.

In conclusion, we have demonstrated tunable positive and negative lenses using PNLC cells. The gradient refractive index is manipulated by a patterned photomask and by controlling the polymer network formation. By using this technique, the operating voltage of the PNLC lens is very low. Moreover, large lens aperture can be fabricated relatively easily.

The authors would like to thank Dr. S. Gauza and Dr. Q.-H. Wang for their kind help in experiments. This work is supported by DARPA under Contract No. DAAD19-02-10208.

${ }^{1}$ S. Sato, J. Appl. Phys. 18, 1679 (1979).

${ }^{2}$ G. D. Love, J. V. Major, and A. Purvis, Opt. Lett. 19, 1170 (1994).

${ }^{3}$ N. A. Riza and M. C. DeJule, Opt. Lett. 19, 1013 (1994).

${ }^{4}$ S. Masuda, S. Takahshi, T. Nose, S. Sato, and H. Ito, Appl. Opt. 36, 4772 (1997).

${ }^{5}$ W. W. Chan and S. T. Kowel, Appl. Opt. 36, 8958 (1997).

${ }^{6}$ A. F. Naumov, M. Yu. Loktev, I. R. Guralnik, and G. Vdovin, Opt. Lett. 23, 992 (1998).

${ }^{7}$ L. G. Commander, S. E. Day, and D. R. Selviah, Opt. Commun. 177, 157 (2000).

${ }^{8}$ J. S. Patel and K. Rastani, Opt. Lett. 16, 532 (1991).

${ }^{9}$ V. V. Presnyako, K. E. Asatryan, and T. V. Galstian, Opt. Express 10, 865 (2002).

${ }^{10}$ H. Ren and S. T. Wu, Appl. Phys. Lett. 81, 3537 (2002).

${ }^{11}$ R. A. M. Hikmet and H. M. J. Boots, Phys. Rev. E 51, 5824 (1995).

${ }^{12}$ T. J. Bunning, L. V. Natarajan, and V. P. Tondiglia, Annu. Rev. Mater. Sci. 30, 83 (2000).

${ }^{13}$ I. C. Khoo and S. T. Wu, Optics and Nonlinear Optics of Liquid Crystals (World Scientific, Singapore, 1993). 